\title{
The non-communicating rudimentary horn: diagnostic and therapeutic challenges
}

\author{
Katrin Scheibner • Annette Kuhn • Luigi Raio • \\ Hermann Brühwiler • Michael D. Müller
}

Received: 13 November 2006 / Accepted: 13 December 2006 / Published online: 16 January 2007

(C) Springer-Verlag 2007

\begin{abstract}
We describe the case of a 23-year-old nulligravid woman who complained of increasing post-menstrual lower abdominal pain. She used contraceptives permanently for three months and was referred with a sub-mucosal lesion suspicious for a type 2 fibroid to be resected. During hysteroscopy, no fibroid mass could be confirmed. A postoperatively performed ultrasound including hydrosonography demonstrated a lesion highly suspicious for a uterus unicollis with a non-communicating uterine horn and a haematometra. Laparoscopy confirmed a normal outer lining of the uterus with hypoplastic tube on the right side. Referring to pre-operative findings, a broadly based uterine horn including a large haematometra was diagnosed. We performed a laparoscopic resection of the rudimentary horn and a right salpingectomy. The patient could be discharged without any complications three days later.
\end{abstract}

Keywords Unicornuate uterus - Rudimentary uterine horn . Congenital mullerian anomaly $\cdot$ Laparoscopy $\cdot$ Haematometra

\section{Introduction}

The prevalence of congenital uterine malformations is about $0.5 \%$. A malformation is a morphologic defect of a body

K. Scheibner $(\bowtie) \cdot$ A. Kuhn $\cdot$ L. Raio $\cdot$ M. D. Müller

Department of Obstetrics and Gynaecology, Inselspital Bern, Effingerstr. 102,

Bern 3010, Switzerland

e-mail: katrin.scheibner@insel.ch

H. Brühwiler

Department of Obstetrics and Gynaecology,

Hospital Münsterlingen,

Münsterlingen, Switzerland region or organ resulting from an intrinsically abnormal developmental process. Reasons for uterine malformations include an incomplete midline unification of the paramesonephric or Mullerian ducts. Depending on the incompleteness of fusion, there is a large variety of malformations. The American Fertility Society (AFS) suggest their specific classification of uterine malformations [1].

A unicornuate uterus is a rare uterine malformation with an incidence of $2.5-13 \%$ [2]. Incomplete fusion of the two Mullerian ducts may lead to a septate or bicornuate uterus; failure of Mullerian tube formation causes aplasia or atresia of one side, resulting in a unicornuate uterus. Incomplete atresia of a Mullerian duct leads to a rudimentary horn which is broadly connected or connected through streak tissue with the unicornuate uterus [3].

A unicornuate uterus may lead to various gynaecological or obstetric complications and diagnostics are often difficult and delayed to the fertile period or to pregnancy. Patients may present with painful menstruation, dyspareunia or malformations of the upper urinary tract, which are frequently inherited with a unicornuate uterus [4]. Rupture of a rudimentary horn is a life-threatening complication in pregnancy $[5,6]$.

We report a case of a unicornuate uterus with a cavitated rudimentary horn which did not communicate and which was misdiagnosed as sub-mucous fibroid and which could have been missed during laparoscopy if sonographic findings had not been acknowledged.

\section{Case report}

A 23-year-old nulligravid women presented with a history of severe dysmenorrhoea since menarche. Oral contraceptives improved the situation slightly. For the past six months, the patient experienced progressive post-menstrual 
pain without any bleeding disorders. Previous endovaginal ultrasound confirmed a hypoechogenic intra-uterine mass of dimensions $2.6 \times 2.8 \mathrm{~cm}$ and it was described as submucous fibroid type 2 (Fig. 1). Both ovaries appeared to be normal. Because of this finding, an oral contraceptive was given continuously for three months. Because the patient showed persisting post-menstrual pain at the followup consultation, she was referred to our unit for hysteroscopic fibroid resection.

Hysteroscopy did not confirm a sub-mucosal fibroid but a normal left uterine cavity with a normal left tubal ostium. The ostium on the right side could not be seen.

Therefore, at the first post-operative day, a vaginal ultrasound including hydrosonography was performed. The uterus on the left was normal with flat endometrium but with fundal broadening. Both ovaries were normal. No findings suspicious for endometriosis could be demonstrated. However, compared to the first appearance of the uterus (Fig. 1), a cavity on the right side now filled with liquid could be demonstrated (Fig. 2a). One could believe that the liquid-filled cavity on the right side results in hysteroscopic insufflation, but, as we described above, this possibility is very unlikely. Grey-scale and power Doppler sonography showed a typical muscular wall with adequate perfusion of this finding. There was no communication towards the left cavity or the cervix, as demonstrated by hydrosonography combined with power Doppler sonography (Fig. 2b-c). Both kidneys appeared to be normal and orthotopic. We suspected a unicornuate uterus with a cavitated noncommunicating rudimentary horn on the right side with haematometra (AFS IIB) and without any signs of major upper urinary tract abnormalities.

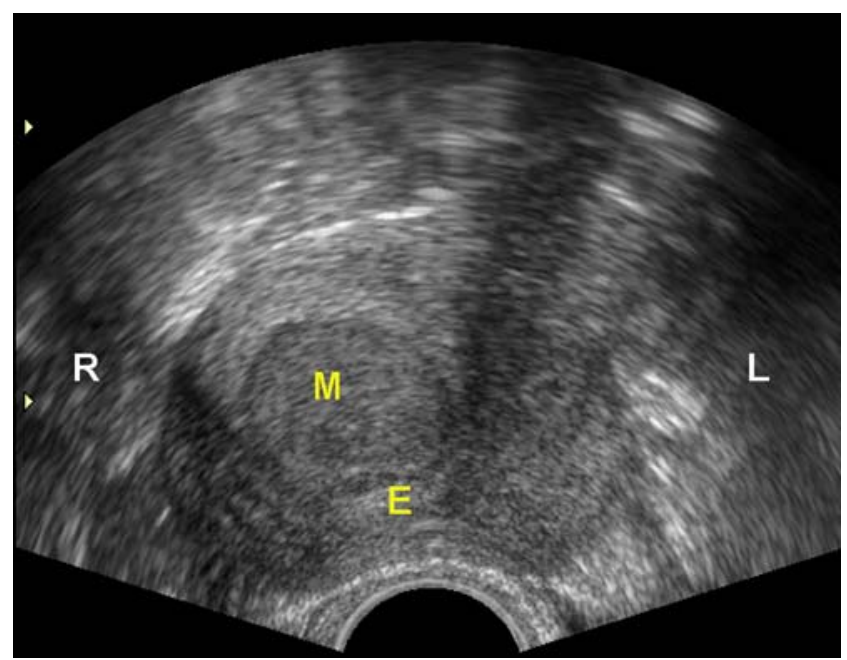

Fig. 1 Initial endovaginal grey-scale ultrasound with a hypoechogenic mass suspicious of being sub-mucous fibroid type $2(M)$, flat endometrium $(E) . R=$ right; $L=$ left
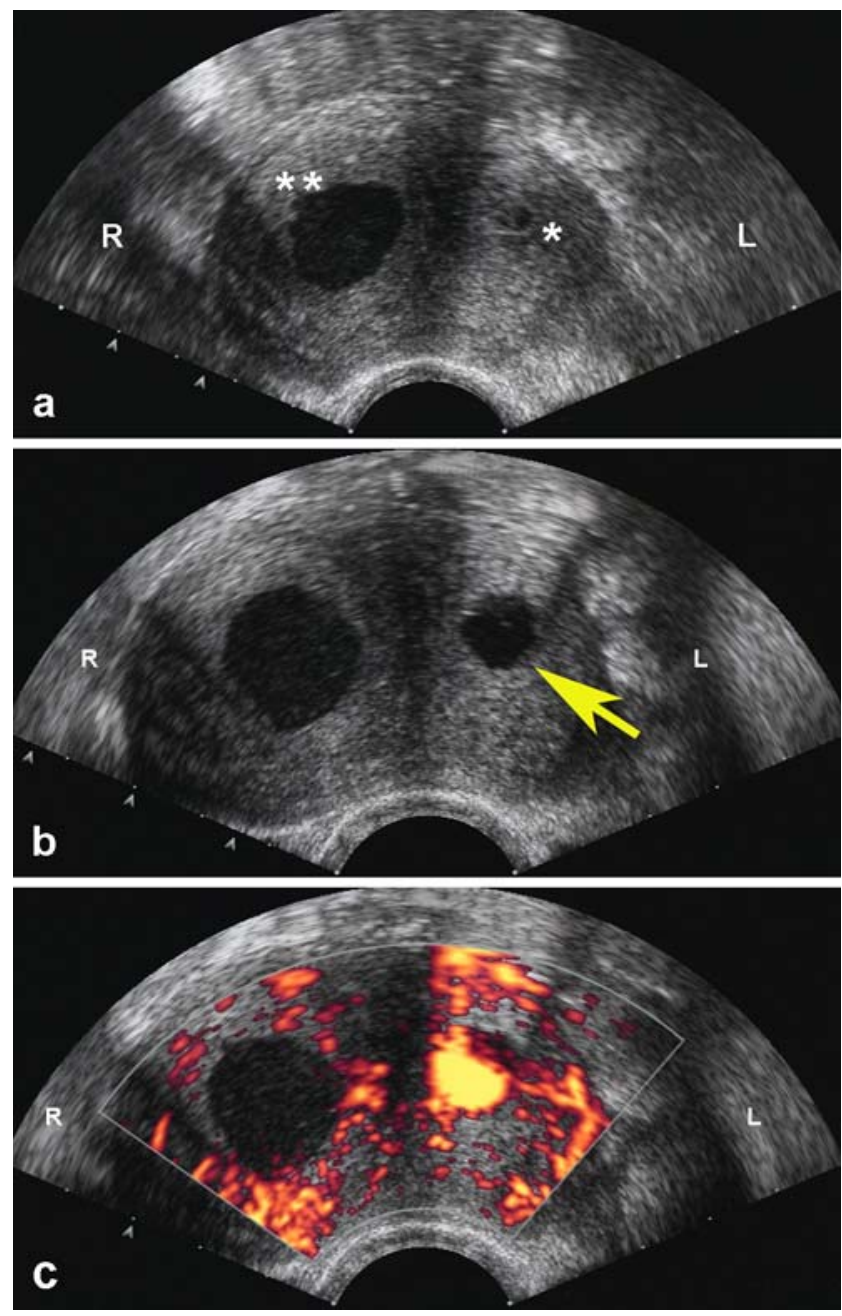

Fig. 2 a Non-communicating rudimentary horn on the right side filled with liquid $(* *)$ and small left cavity before hydrosonography $(*)$. b Note the filling of the left cavity during hydrosonography (arrow), while the right cavity did not show any changes. c This could be confirmed by power Doppler sonography, where turbulent flow was detected during injection of saline only in the left uterine cavity. $R=$ right; $L=$ left

The patient underwent laparoscopy using one infraumbilical and three suprapubic accessory trocars under general anaesthesia. The anatomic findings were as follows:

- The uterus was slightly enlarged with normal left round ligament

- The ovary and the fallopian tube on the left appeared to be normal

- On the right, there was a hypoplastic fallopian tube, particularly of the isthmic area with normal fimbriae

- The right ovary was atrophic

- There were neither signs of endometriosis nor cul-desac obliteration

- The uterine horn on the right was enlarged and attached to the unicornuate uterus at full length

- It was very difficult to distinguish the planes between the two horns (Fig. 3) 


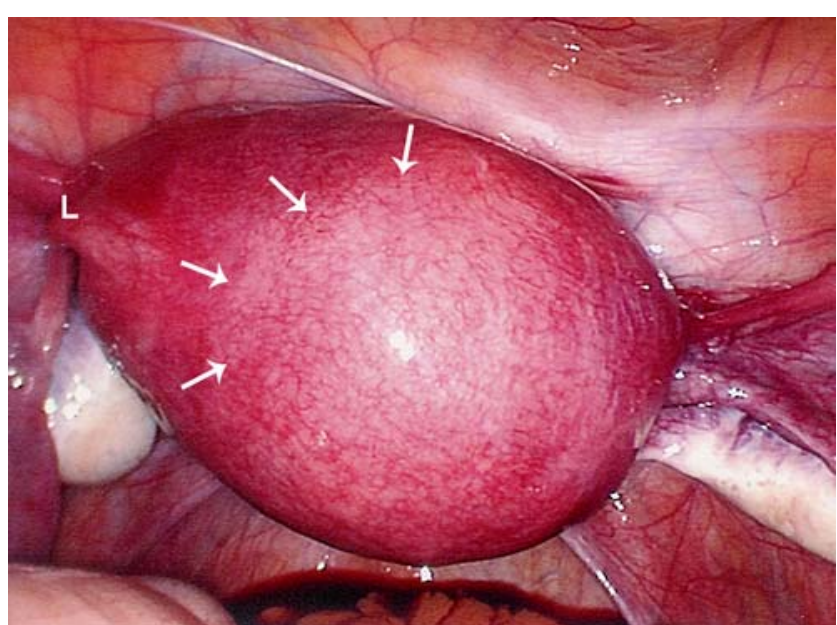

Fig. 3 Intra-operative findings: uterus with blind, broadly attached uterine horn and a hypoplastic fallopian tube on the right. Greater difficulty to distinguish the planes between the two horns (arrows). $L=$ left

The round ligament, isthmic area of the fallopian tube and utero-ovarian ligament ipsilateral to the rudimentary horn were coagulated and separated. We entered the retroperitoneal space to identify the ureters. The upper branch of the uterine artery was coagulated and separated. Diluted adrenalin solution $(0.1 \mathrm{mg}$ noradrenalin in $20 \mathrm{ml}$ $\mathrm{NaCl}$ ) was injected into the rudimentary horn. At the attachment site of the rudimentary horn, the rudimentary cavity was primarily opened and then the myometrium was transsected using coagulation (Fig. 4). The haematometra was identified and the cavity was marked with methylene blue. The rudimentary horn was resected. The large defect of the myometrium was closed with two layers of interrupted sutures and the round ligament on the right was reattached to the uterus (Fig. 5). The fallopian tube on

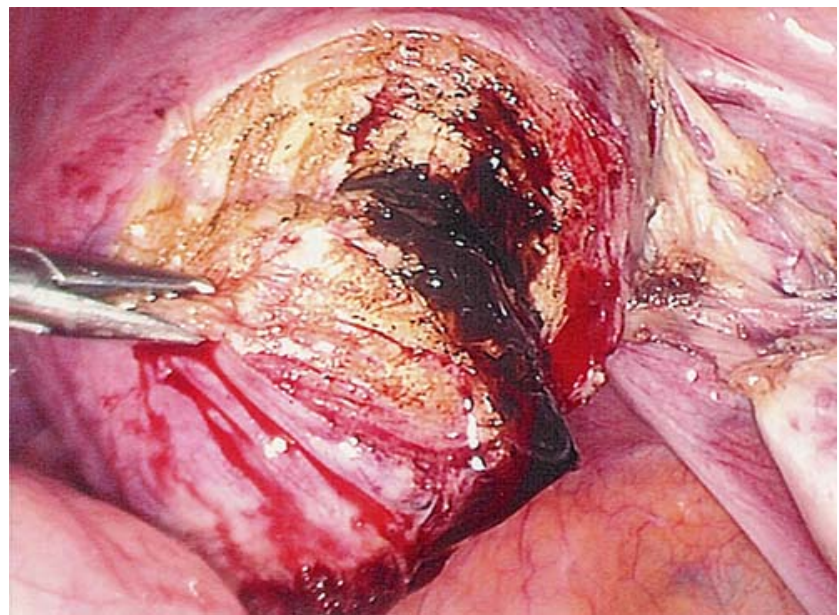

Fig. 4 After the opening of the rudimentary horn, haematometra was identified

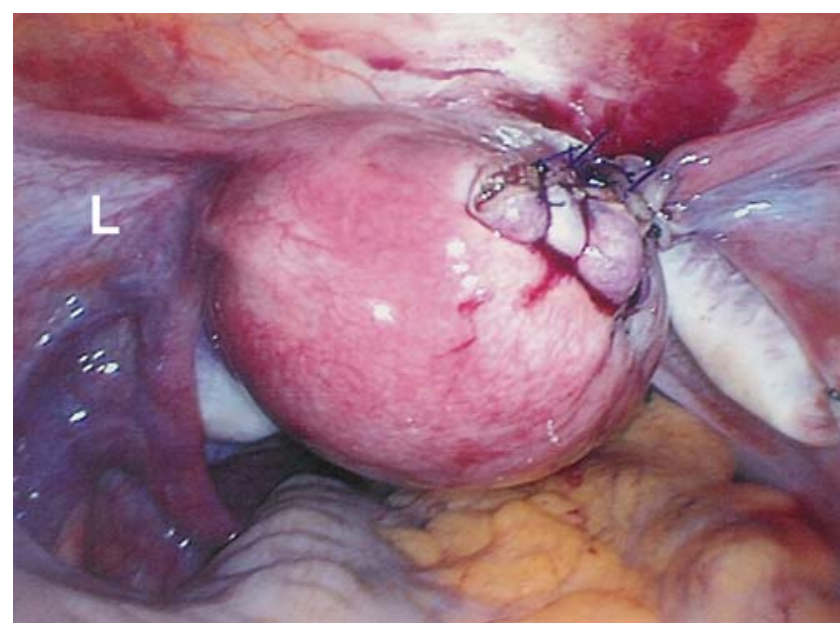

Fig. 5 Uterus after resection of the right horn. $L=$ left

the left was also excised. The right ovary could be saved. The rudimentary horn was removed through a posterior colpotomy. The post-operative period was uneventful and the patient was discharged on the third post-operative day. Pathology confirmed thickened myometrium, partly including atrophic endometrium with pseudo-decidualisation of the stroma, as well as adenomyosis uteri and siderophages. The right fallopian tube was without pathological findings.

\section{Discussion}

A unicorn uterus with a rudimentary horn is a rare uterine malformation, which explains why most general gynaecologists have little experience with this disease.

Because of the lack of experience and incorrect interpretation of clinical and ultrasound findings, diagnosis is difficult and often found by chance only when the patient presents with sterility or complications [6]. As the literature demonstrates, early diagnosis is of great importance in order to avoid consecutive damage of the reproductive system and further painful complications $[4,7,8]$.

To get to the correct diagnosis, the patient's history is crucial: increasingly painful periods, dyspareunia and sterility are common symptoms [2, 8, 9]. Our patient complained of increasing post-menstrual pain, which, in retrospective, we consider to be due to the increasing size of the rudimentary horn.

To obtain the proper diagnosis, endovaginal grey-scale sonography is mandatory. As an additional method to investigate congenital abnormalities of the uterus, Salle et al. recommended hydrosonography [10]. Three-dimensional ultrasound and magnetic resonance imaging (MRI) may also give additional information, as well as hysteroscopy [2]. Often, laparoscopy is able to lead to the exact diagnosis of a uterine malformation [11]. 
The classification of uterine malformations is described by the ASF [1]. A unicorn uterus with a rudimentary horn is rather rare; case-control studies have demonstrated that rudimentary horns are mainly right-sided, as in our patient, which is the case in about $60 \%$ of patients $[5,12]$. There is no evident explanation for this finding. Usually, the ipsilateral ovary is of normal function, as it is not of Mullerian duct origin but may be abnormally located. Extra pelvic localisations have been described [5].

If undetected, a unicorn uterus with a non-communicating cavitated horn is associated with a high incidence of complications. The fact that haematometra, haematosalpinx or even endometriosis are consequences of retrograde menstruation has already been described. The reason our patient did not develop endometriosis and only showed haematometra could be explained by the existence of a hypoplastic right tube that prevented retrograde menstruation.

The diagnosis and appropriate treatment of a rudimentary horn should be carried out prior to pregnancy. Indeed, several complications have been described in the literature. Tubal pregnancy located in the tube near the rudimentary horn might be due to intra-peritoneal sperm or oocyte migration [2, 13]. Even pregnancies in the rudimentary horn have been described and show a higher incidence for abortion and rupture of the horn, particularly in the second or third trimester. The reason for uterine rupture is either due to a thinned muscular wall or an unphysiological implantation. This is why the rudimentary horn and the connecting tube should be removed in the course of a therapeutic laparoscopy [5].

A sub-mucosa fibroid was suspected, although the patient did not complain of any bleeding disorders. The differential diagnosis was adenomyosis. In the initial ultrasound, we wrongly interpreted the hypoechogenic mass caused by thickened menstrual blood as intra-uterine fibroid. Long-term oral contraceptive did not improve the pain and surgery was suggested.

Against expectations, the hysteroscopy showed only one tubal ostium with a normal uterine cavity. The endovaginal ultrasound carried out after hysteroscopy showed a indubitably liquid-filled second cavity that we interpreted as being a liquefaction of the haematometra, possibly due to the use of long-term oral contraceptives. After the additional use of hydrosonography, we suspected the existence of a unicorn uterus with a rudimentary horn. These malformations are, in $30-40 \%$ of cases, associated with further urogenital malformations. Therefore, we performed an MRI, which did not show any pathological findings of the kidneys or ureters [4].

Without the thorough knowledge of the pre-operative findings, we could have missed the uterine malformation, which has been described in the literature before, despite the experienced surgeons [7]. A detailed pre-operative diagnosis is crucial for the surgeon, as the removal of the rudimentary horn might be technically difficult: the layer between the rudimentary horn and the uterus is often barely visible $[3,8]$. We used indigo carmine to dye the cavum of the rudimentary horn, which then was removed with a triangular excision without damaging the uterus. Case reports have described the use of hysteroscopic luminoscopy or intra-operative ultrasound with the use of specific transducers [14].

To avoid excessive bleeding during removal of the rudimentary horn, the knowledge of arterial blood supply is essential. Not only the ipsilateral uterine artery but also contralateral arcuate arteries inside the myometrium supply the rudimentary horn, which require coagulation $[5,6]$. Finally, after the removal of the rudimentary horn, the muscular defect has to be closed, for which, the laparoscopic technique needs to be elaborated [15].

\section{Conclusion}

Particularly in pre-menopausal patients who present with abdominal pain, adnexal masses of unknown origin and severely painful periods without any signs of endometriosis, we must consider an anomaly of the Mullerian duct. Early diagnosis of a uterine malformation is essential to prevent complications. Pre-operative diagnosis is of great importance to enable adequate treatment to be undertaken. Such operations should only be performed by experienced surgeons with a high level of technical skill.

\section{References}

1. American Society for Reproductive Medicine (ASRM) (1988) The American Fertility Society classifications of adnexal adhesions, distal tubal occlusion, tubal occlusion secondary to tubal ligation, tubal pregnancies, Mullerian anomalies and intrauterine adhesions. Fertil Steril 49(6):944-955

2. Chakravarti S, Chin K (2003) Rudimentary uterine horn: management of a diagnostic enigma. Acta Obstet Gynecol Scand 82(12):1153-1154

3. Falcone T, Gidwani G, Paraiso M, Beverly C, Goldberg J (1997) Anatomical variation in the rudimentary horns of a unicornuate uterus: implications for laparoscopic surgery. Hum Reprod 12 (2):263-265

4. Jayasinghe Y, Rane A, Stalewski H, Grover S (2005) The presentation and early diagnosis of the rudimentary uterine horn. Obstet Gynecol 105(6):1456-1467

5. Heinomen PK (1997) Unicornuate uterus and rudimentary horn. Fertil Steril 68(2):224-230

6. Samuels TA, Awonuga A (2005) Second-trimester rudimentary uterine horn pregnancy: rupture after labor induction with misoprostol. Obstet Gynecol 106(5 Pt 2):1160-1162

7. Dimitrova V, Nalbanski B (1997) The echographic diagnosis of a rare congenital uterine anomaly (uterus unicornis with a rudimentary noncommunicating horn) (in Bulgarian). Akush Ginekol (Sofia) 36(2):44-47 
8. Kriplani A, Agarwal N (2001) Hysteroscopic and laparoscopic guided miniaccess hemihysterectomy for non-communicating uterine horn. Arch Gynecol Obstet 265(3):162-164

9. Atmaca R, Germen AT, Burak F, Kafkasli A (2005) Acute abdomen in a case with noncommunicating rudimentary horn and unicornuate uterus. JSLS 9(2):235-237

10. Salle B, Sergeant P, Gaucherand P, Guimont I, de Saint Hilaire P, Rudigoz RC (1996) Transvaginal hysterosonographic evaluation of septate uteri: a preliminary report. Hum Reprod 11(5):1004-1007

11. Takeuchi H, Sato Y, Shimanuki H, Kikuchi I, Kumakiri J, Kitade M, Kinoshita K (2006) Accurate preoperative diagnosis and laparoscopic removal of the cavitated non-communicated uterine horn for obstructive Mullerian anomalies. J Obstet Gynaecol Res 32(1):74-79
12. Fedele L, Bianchi S, Zanconato G, Berlanda N, Bergamnini V (2005) Laparoscopic removal of the cavitated noncommunicating rudimentary uterine horn: surgical aspects in 10 cases. Fertil Steril 83(2):432-436

13. Handa Y, Hoshi N, Yamada H, Wada S, Kudo M, Tsuda K, Sagawa T, Fujimoto $S$ (1999) Tubal pregnancy in a unicornuate uterus with rudimentary horn: a case report. Fertil Steril 72(2):354-356

14. Tanaka Y, Asada H, Uchida H, Maruyama T, Kuji N, Sueoka K, Yoshimura Y (2005) Case of iatrogenic dysmenorrhea in noncommunicating rudimentary uterine horn and its laparoscopic resection. J Obstet Gynaecol Res 31(3):242-246

15. Nezhat CR, Smith KS (1999) Laparoscopic management of a unicornuate uterus with two cavitated, non-communicating rudimentary horns: case report. Hum Reprod 14(8):1965-1968 